\title{
Development and Implementation of an Antimicrobial Stewardship Program in a Rural Hospital
}

\author{
Kerry-Anne Hogan, Mohamed Gazarin, and Julie Lapenskie
}

\section{INTRODUCTION}

Tn Canada, antimicrobial stewardship is a Required Organizational Practice for accreditation of hospitals. ${ }^{1}$ Given long-standing misuse of antimicrobials, systematic, multifaceted, and proactive interventions to combat resistance and preserve currently available antimicrobials are imperative. ${ }^{2}$ Antimicrobial stewardship programs (ASPs) have shown success in reducing antimicrobial use, mitigating antimicrobial resistance, and improving patient outcomes and cost-effectiveness. ${ }^{2-4}$

The Winchester District Memorial Hospital is a 49-bed teaching hospital in Winchester, Ontario, with a small in-house pharmacy department employing 2 full-time clinical pharmacists. The pharmacy department is open weekdays from 0800 to 1600 and processes about 100 prescriptions per day for the maternal-child, medical-surgical, chemotherapy, extended care, and operating room services. In July 2012, with limited resources, no funding, and a wide spectrum of existing responsibilities, one of the pharmacists (M.G.) established a multidisciplinary team, on the basis of recommendations from Accreditation Canada, ${ }^{1}$ to develop, implement, and oversee an ASP. The ASP team consists of a hospital-based general practitioner, a pharmacist, an infection control nurse, a staff nurse, a hospital administrator, and an information technologist. Two partners from other organizations (the infectious disease specialist responsible for the region and a microbiologist) provide support and direction as required.

Because of limited in-house expertise, the ASP team relied on the published literature and inquiries to other facilities for guidance. The paucity of literature on ASPs in rural hospitals represented a significant barrier to development and initiation of the program. The existing literature is largely irrelevant to the setting of the Winchester District Memorial Hospital because most of the hospitals described are substantially larger and have more resources, particularly with regard to the quan- tity and specialization of staff. As a result, the team had to rely on external expertise for direction and mentoring on the typical components of an ASP and for pathogen- and antimicrobialspecific recommendations. The in-house stewardship team then adapted the components of established ASPs to suit the hospital's needs. Development and implementation of this program took about $35 \mathrm{~h}$, and ongoing management requires $1.5 \mathrm{~h} /$ day on the part of the pharmacist. The ASP physician is an on-staff general practitioner, with $1 \mathrm{~h}$ of time dedicated to the program each week. A mean of 20 patient charts are audited daily, and on average 6 of these patients are receiving antimicrobial treatment. Two or three cases per day require intervention from the pharmacist to suggest adjustment of therapy, with each case requiring 10-30 min to complete.

Through the literature review of ASPs, ${ }^{5-7}$ the team decided on 7 program objectives (Box 1), which in turn provided the impetus to develop strategies for success. These strategies are present in many ASPs; they have been effective in reducing rates of antimicrobial resistance, use, and $\operatorname{cost}^{8}$; and they involve the participation of all clinical staff. The team decided to promote antimicrobial stewardship as a responsibility of all clinical staff, with a focus on improving outcomes related to antimicrobial use in a resource-poor setting such as a rural community.

\section{Box 1. Goals of the Winchester District Memorial} Hospital Antimicrobial Stewardship Program

Advance the knowledge and skills of primary care providers in prescribing antimicrobials

Optimize clinical outcomes of patients being treated with antimicrobial agents

Enable more cost-effective antimicrobial prescribing practice Develop a culture of antimicrobial stewardship within the facility Reduce the risk of infections

Reduce or stabilize levels of antibiotic resistance Promote patient safety 


\section{DESCRIPTION OF THE ASP}

The implementation and success of the ASP have been highly dependent upon adherence to the predefined strategies. Although the primary responsibility for stewardship falls to the prescriber and the pharmacist, the culture of our organization values the contribution of the nursing team, and nurses have been engaged as active members of the ASP team in reducing the misuse of antibiotics. The 7 strategies consist of 2 core strategies (conducting a prospective audit with intervention and feedback and enforcing formulary restriction and the need for authorization for nonformulary antibiotics) and 5 supplemental strategies (education, availability of guidelines, use of clinical pathways, escalation and de-escalation of therapy, and encouragement of IV-to-oral [PO] conversion).

\section{Core Strategies}

\section{Conduct a Prospective Audit with Intervention and Feedback}

Before implementation of the ASP, the pharmacist performed a general review of antimicrobial orders to detect discrepancies (with respect to clinical guidelines) with individual antimicrobial agents, including indication, dose, frequency (in relation to renal function), and duration of therapy. Since inception of the ASP, the pharmacist now reviews each medication order by physically reviewing every patient's chart (manually or electronically, if available) to determine the condition for which the patient is being treated and the medical history. These reviews include antibiotics given by the IV route, with particular attention to ceftazidime, ciprofloxacin, meropenem, piperacillin-tazobactam, and vancomycin. If an order is found to be inappropriate or suboptimal, the pharmacist contacts the prescriber before dispensing the medication, in an effort to adjust therapy to an antimicrobial that is more specific and individualized to the patient case.

For example, it was commonly found that piperacillintazobactam was prescribed for patients with severe lung infections but no risk factors for Pseudomonas aeruginosa; similarly, vancomycin was prescribed for severe cellulitis in patients with peripheral arterial disease and no risk factors for methicillin-resistant Staphylococcus aureus (MRSA). In both scenarios, a de-escalation approach was adopted, which allowed for the use of narrow-spectrum antibiotics, such as ceftriaxone or ertapenem, and replacement of vancomycin with cefazolin plus appropriate non-drug measures, such as elevating the limb to control edema and using a skin emollient. This approach increased the visibility of the pharmacist in terms of his infectious disease expertise, led to greater utilization of pharmacy consultations for antimicrobial selection and renal dosing, enhanced collaborative patient care, and ensured safe prescribing practices.

\section{Enforce Formulary Restriction and Need for} Authorization for Nonformulary Antibiotics

Formulary restriction and preauthorization are considered to form the most effective approaches to controlling the use of antimicrobial agents ${ }^{9}$; at the Winchester District Memorial Hospital, formulary-related decisions are made by the Pharmacy and Therapeutics Committee. Formulary restriction refers to limiting a facility's antimicrobial formulary according to factors such as efficacy, toxicity, cost, and redundancy. For example, linezolid, an antibiotic prescribed for vancomycinresistant enterococcus and multidrug-resistant strains, is no longer on the hospital's formulary but can be ordered on a case-by-case basis. If a nonformulary antibiotic is prescribed, the prescriber must provide justification for its use, and the drug must be authorized by the ASP pharmacist. If determined to be appropriate, the antimicrobial is ordered from a neighbouring tertiary care facility (The Ottawa Hospital) according to the dose and duration of therapy for the specific case.

In the event that the pharmacist and the physician do not agree on the best therapy options for a particular case (which occurs on average twice a month), the infectious disease physician is consulted. The ability to consult with external experts, such as the infectious disease physician, is an important component of the sustainability of rural hospitals. Larger tertiary care facilities have many specialists and, through networking and regionalization, are usually willing to share their expertise with smaller hospitals like the Winchester District Memorial Hospital.

With the limited formulary and a desire to have a pharmacist review the appropriateness of orders before dispensing, antibiotics are not kept in clinical departments. When the pharmacist is not on duty, the prescribing physician can order the first doses of an antimicrobial, with the charge nurse being the only person who can retrieve the antibiotic from the locked pharmacy; once the pharmacist returns, he reviews the orders and makes recommendations as required. This practice helps to reduce costs and controls the dispensing of antimicrobials, to ensure that each patient receives the best recommended treatment.

Because of limited pharmacy hours, the lack of an on-site infectious disease physician, and the high cost of specific and broad-spectrum antibiotics, the ASP team wanted to limit the number of antibiotics on formulary, choosing not to add linezolid, daptomycin, or fidaxomycin. Prescribers are thus limited to choosing IV antibiotics that are on formulary, including the following 5 broad-spectrum antibiotics: ceftazidime, ciprofloxacin, meropenem, piperacillin-tazobactam, and vancomycin. When one of these antimicrobials is prescribed, a reminder formulary label outlining antibioticspecific conditions of use, best practice prescribing guidelines for the antibiotic, and antimicrobial alternatives is printed and sent to the ward with the patient's medications. The informa- 
tion on the label for each of these drugs was developed by the pharmacist and the ASP physician on the basis of evidence and practices from The Ottawa Hospital, and the content of each label has been approved by the infectious disease physician.

Although there have been instances when a label was not appropriately placed in a patient's chart, the larger barrier to implementing this labelling practice was initial resistance from physicians, who saw the label as a directive rather than a recommendation. With time, collaboration, and positive feedback, the physicians and the pharmacist have come to recognize this practice as an opportunity for communication and learning, with the ultimate goal being patient safety.

As an alternative to printing a reminder label when one of the broad-spectrum antibiotics is prescribed, the pharmacist may explore the treatment choice through direct discussions with the prescriber and may recommend a different antibiotic. Physicians' cooperation and interest in supporting the goals of the ASP have prevented the need to enforce formulary restrictions. Having physician support has led to improved prescribing for ciprofloxacin, piperacillin-tazobactam, and vancomycin, whereby the appropriate drug is prescribed for the correct indications of use, rather than a particular antimicrobial being selected "just in case" (ceftazidime and meropenem were typically prescribed appropriately even before the ASP was developed and implemented). However, there is a lack of objective supporting data, as data were not collected consistently during implementation of the program.

\section{Supplemental Strategies}

The Winchester District Memorial Hospital is committed to enhancing the ASP across all disciplines. This objective has been achieved by embedding supplemental strategies into the 2 core strategies described above. The following feasible and sustainable strategies were chosen: providing education, making up-to-date guidelines available, using clinical pathways, performing escalation and de-escalation of therapy as appropriate, and making conversion recommendations. The strategies were developed by the Pharmacy and Therapeutics Committee and are based on recommendations from Accreditation Canada ${ }^{1}$ and Public Health Ontario. ${ }^{7}$

\section{Education}

For a 2-month period before implementation of the ASP, all clinical staff received education highlighting the importance of and their role in the ASP and the use of guidelines; this education also emphasized the long-term negative effects of not having an ASP, which include increases in antibiotic resistance, expenditures, IV use, and infections. The lead pharmacist (M.G.), in consultation with the ad hoc infectious disease specialist, provided initial education to staff on antimicrobial stewardship, clinical practice guidelines, and the hospital's ASP. Physician education was provided by the ASP team's physician during meetings of the Pharmacy and Therapeutics Committee, the Medical Advisory Committee, and the Medical Staff Organization. The education sessions continued for about 1 month after ASP implementation, and now, continuing education sessions are offered quarterly by the external infectious disease specialist; although these sessions are primarily targeted toward physicians, any staff member may attend. Topics are selected on the basis of staff requests and availability of external expertise, and have included antimicrobial stewardship, skin and soft-tissue infections, management of pneumonia, management of symptomatic and asymptomatic urinary tract infections, antibiogram analysis and subsequent decisionmaking, and sepsis. The infection control nurse, clinical education nurse, and charge nurses are responsible for conveying relevant information to all staff nurses. The education sessions are well attended, and anecdotal reports suggest that they are beneficial; in addition, evidence of practice changes after the sessions has been observed.

\section{Accessible Guidelines}

The Canadian-based Anti-infective Guidelines for CommunityAcquired Infections ${ }^{10}$ and The Sanford Guide to Antimicrobial Therapy $^{11}$ are available (as hard copies) on patient care units for clinical staff to use as references. Both of these guideline documents provide recommendations for drug choice; although the hospital uses primarily the Canadian guidelines, the Sanford guide is beneficial for more complex cases. Before implementation of the ASP, these references were inconsistently available.

\section{Use of Clinical Pathways}

Rural-friendly clinical pathways are used to structure patient care activities for community-acquired pneumonia, febrile neutropenia, influenza prophylaxis and treatment, sepsis and bacteremia, urinary tract infections, and surgical care. These pathways, which include nursing care plans, patient information, and preprinted orders, are available to staff members through the hospital's intranet, and the pertinent care pathway is printed for each patient according to diagnosis. This print-as-needed approach allows for updates to be made in the electronic version as guidelines evolve and prevents the use of out-of-date forms. Stewardship processes are embedded in these pathways through clinical guidelines and information from partnering hospitals. The pathways are reviewed annually by the ASP team and updated as required.

\section{Escalation and De-escalation of Therapy}

Empiric therapy usually begins in the absence of a culture and sensitivity report on the causative pathogen and is thus based on clinical discretion. This approach sometimes results in the use of relatively broader-spectrum antimicrobials than 
are necessary, but antimicrobial stewardship dictates that therapy be tailored to the causative pathogen when possible; this was the impetus for limiting the drug formulary. Escalation or de-escalation of therapy is a collaborative responsibility among the attending physician, the laboratory department, and the pharmacist. The pharmacist's role is to ensure that therapy is modified (made narrower or broader) or discontinued (if a bacterial pathogen is unlikely), on the basis of serum antimicrobial level and culture and sensitivity results. Every antimicrobial order is reviewed for opportunities to optimize therapy, and susceptibility results are available through the daily report of culture results generated by the laboratory and printed by the pharmacist. Recommendations based on these results are communicated by speaking with the prescribing physician directly and documenting the information in the patient's progress notes.

\section{IV-to-PO Step-Down Conversion}

The ASP team was responsible for developing a process for IV-to-PO step-down conversion, and used 3 main strategies in doing so: education, surveillance, and case review. Education about the criteria for IV-to-PO conversion and adherence with best practice and current clinical guidelines was provided to physicians and nurses during implementation of the ASP and is now reinforced quarterly. Nurses are instructed to notify pharmacy staff when any IV antimicrobial has exceeded the recommended duration of therapy, based on clinical guidelines and patient-specific eligibility for IV-to-PO step-down. Physician education focuses on building awareness of IV-toPO step-down recommendations. The pharmacy department monitors records of all IV orders so that the pharmacist can assess the need for IV administration and contact the physician to suggest a PO antimicrobial alternative, if appropriate. In noncritical instances (determined by how close a patient is to being eligible for IV-to-PO step-down), a label suggesting PO step-down choices and criteria to justify the conversion is provided and affixed to the progress notes. Three days after making a step-down recommendation, the pharmacist initiates a case review and, if necessary, contacts the physician to determine why the conversion has not been carried out. This process ensures that the route change has been considered and provides an educational opportunity for both parties.

\section{PROGRAM DEVELOPMENT AND IMPLEMENTATION CHALLENGES}

There were several barriers to development and implementation of the Winchester District Memorial Hospital ASP. Initially, there was a general lack of understanding among staff about ASPs. Staff members who were interested in ASPs did not know how to develop one and were unsure who should be leading such a program, because the hospital lacked an infectious disease specialist. Overcoming this first development barrier involved selecting an individual to take ownership of and spearhead the ASP. The second barrier was the lack of funding and time, and members of the team had to be willing to work overtime without compensation.

Other barriers to program implementation included lack of consistent education to all staff on ASP practices, lack of adequate buy-in and uptake by physicians, and lack of role clarity about the responsibility for antimicrobial prescribing among disciplines. For example, the traditional nursing role is merely administration of medications, but within the ASP, nurses are expected to "police" the prescribing process and to notify the pharmacist about potential violations. Regular and positive communication among staff members, particularly between physicians and the ASP pharmacist, and empowerment of nurses have been essential to the success of the ASP.

\section{Evaluation}

The effectiveness of the ASP has been measured primarily through changes in susceptibility to targeted antimicrobials among various gram-negative and gram-positive organisms (Table 1).Over time, the susceptibility rates for commonly used antibiotics have shown either a positive trend or have remained stable.

The ASP has also been evaluated in terms of the number of pharmacist recommendations made and the number accepted. Antimicrobial recommendations made (and tracked) by the ASP pharmacist increased from an average of 2.5 per week upon initiation of the ASP (in 2012) to 15 per week in December 2014, with a weekly average of $13(87 \%)$ of the 15 recommendations being accepted. Over the same period, pharmacy requests for infectious disease consults increased from 1 per month to 3 per week.

Consumption of targeted IV antibiotics, defined daily doses, and antimicrobial acquisition costs were also measured. Among the targeted IV antibiotics, piperacillin-tazobactam and vancomycin usage was reduced the most, with the defined daily dose per 1000 patient-days decreasing from 21.36 to 17.54 for piperacillin-tazobactam and from 18.99 to 12.21 for vancomycin. There were overall trends for reductions in consumption, defined daily dose, and cost, but the relationship between these trends and the ASP could not be substantiated because of multiple demographic and organizational changes during the implementation phase, including increases in the numbers of patients requiring antibiotics and hospital admissions, a reduction in the length of hospital stay, and a lack of alternative level of care patients.

It is difficult to ascertain which ASP strategies resulted in the changes discussed above. We believe that substituting a narrow-spectrum antibiotic for piperacillin-tazobactam, targeting the overall utilization of vancomycin, and increased awareness of risk factors for MRSA played significant roles in the reduction of these antibiotics. Because all of the 
This single copy is for your personal, non-commercial use only.

For permission to reprint multiple copies or to order presentation-ready copies for distribution, contact CJHP at cjhpedit@cshp.ca

Table 1. Antibiogram for the Winchester District Memorial Hospital, 2011-2014*

Medication; \% Susceptibility†

\begin{tabular}{|c|c|c|c|c|}
\hline \multirow[b]{2}{*}{ Organism‡ } & \\
\hline & Ciprofloxacin & Piperacillin-Tazobactam & Meropenem & Ceftazidime \\
\hline \multicolumn{5}{|l|}{ Gram-negative } \\
\hline \multicolumn{4}{|l|}{ Escherichia coli } & NA \\
\hline $2011(n=337)$ & 91 & 92 & 100 & \\
\hline $2012(n=309)$ & 81 & 96 & 100 & \\
\hline $2013(n=333)$ & 86 & 94 & 100 & \\
\hline $2014(n=335)$ & 84 & 96 & 100 & \\
\hline \multicolumn{4}{|l|}{ Klebsiella pneumoniae } & NA \\
\hline $2011(n=45)$ & 100 & 97 & 100 & \\
\hline $2012(n=47)$ & 97 & 97 & 100 & \\
\hline $2013(n=43)$ & 100 & 97 & 100 & \\
\hline $2014(n=42)$ & 98 & 95 & 100 & \\
\hline \multicolumn{5}{|l|}{ Pseudonomas aeruginosa } \\
\hline $2011(n=25)$ & 84 & 92 & 96 & 92 \\
\hline $2012(n=41)$ & 75 & 92 & 90 & 92 \\
\hline $2013-2014(n=43)$ & 86 & 95 & 93 & 95 \\
\hline \multicolumn{5}{|l|}{ Gram-positive } \\
\hline Methicillin-sensitive & Clindamycin & Cefazolin & Cloxacillin & \\
\hline \multicolumn{5}{|l|}{ Staphylococcus aureus } \\
\hline $2011(n=79)$ & 79 & 100 & 100 & \\
\hline $2012(n=103)$ & 79 & 100 & 100 & \\
\hline $2013(n=121)$ & 81 & 100 & 100 & \\
\hline $2014(n=88)$ & 86 & 100 & 100 & \\
\hline \multicolumn{5}{|l|}{ Staphylococcus aureus } \\
\hline $2011-2012(n=33)$ & 48 & 97 & & \\
\hline $2013-2014(n=39)$ & 54 & 97 & & \\
\hline Streptococcus pneumoniae & Erythromycin & Levofloxacin & & \\
\hline $2011(n=11)$ & 91 & 91 & & \\
\hline $2012(n=8)$ & 63 & 100 & & \\
\hline $2013(n=5)$ & 80 & 100 & & \\
\hline $2011-2014(n=28)$ & 79 & 96 & & \\
\hline
\end{tabular}

NA = not applicable.

* Data provided by the microbiology department of The Ottawa Hospital.

+Percent susceptibility is given for target antimicrobials of selected gram-negative and gram-positive isolates.

\#For each year, the number of isolates tested for each organism is shown in parentheses.

interventions were implemented simultaneously, it is not possible to delineate specifically which strategies were and were not effective. We suggest that, to allow determination of which interventions are most effective, future programs should start small, implementing just 1 or 2 strategies before adding others, and should define outcome measures at the outset of program development, to allow measurement of the impact of an intervention after its implementation. This approach would allow for more comprehensive data collection, improved program evaluation, and potential reduction in resistance from staff members who prefer small, incremental changes in daily practice.

\section{CONCLUSION}

The Winchester District Memorial Hospital is a leader in antimicrobial stewardship and an exemplary case of how a small (49-bed) hospital was able, with minimal resources, to mobilize and implement a successful ASP by overcoming barriers unique to the rural setting. Evidence-based practice guidelines clearly highlight the need for and benefit of antimicrobial stewardship within health care institutions; however, the mere publication of recommendations has not led to the widespread implementation of guidelines in hospitals. The Winchester District Memorial Hospital represents the forefront of change by moving research into practice, employing locally adapted, evidence-based practice standards in an efficient manner to promote a cultural shift in prescribing behaviour. This program will ultimately improve outcomes for patients through the avoidance of antimicrobial resistance and adverse effects and the preservation of the current antimicrobial armamentarium. This article serves as evidence supporting comprehensive ASPs and their facilitation through minimal resources in a small, rural hospital. 
References

1. Required organizational practices: handbook 2016. Ottawa (ON): Accreditation Canada; 2015 [cited 2016 Feb 18]. Available from: https:// accreditation.ca/sites/default/files/rop-handbook-2016-en.pdf

2. Dellit TH, Owens RC, McGowan JE, Gerding DN, Weinstein, RA, Burke JP, et al. Infectious Diseases Society of America and the Society for Healthcare Epidemiology of America guidelines for developing an institutional program to enhance antimicrobial stewardship. Clin Infect Dis. 2007;44(2):159-77.

3. Fishman N. Antimicrobial stewardship. Am J Infect Control. 2006; 34(5 Suppl 1):S55-63.

4. Kaki R, Elligsen M, Walker S, Simor A, Palmay L, Daneman N. Impact of antimicrobial stewardship in critical care: a systematic review. $J$ Antimicrob Chemother. 2011;66(6):1223-30.

5. Antimicrobial stewardship toolkit: best practices from the GNYHA/UHF Antimicrobial Stewardship Collaborative. New York (NY): Greater New York Hospital Association and United Hospital Fund; 2011 [cited 2015 June 22]. Available from: www.shea-online.org/Portals/0/GNYHA_ Antimicrobial_Stewardship_Toolkit_FINALv2\%20Dec2011.pdf

6. Duguid M, Cruickshank M, editors. Antimicrobial stewardship in Australian hospitals. Sydney (Australia): Australian Commission on Safety and Quality in Health Care; 2011 [cited 2015 June 22]. Available from: www.safetyandquality.gov.au/wp-content/uploads/2011/01/ Antimicrobial-stewardship-in-Australian-Hospitals-2011.pdf

7. Ontario Agency for Health Protection and Promotion (Public Health Ontario). Getting started: a gap analysis tool for antimicrobial stewardship programs. Toronto (ON): Queen's Printer for Ontario; 2016 [cited 2016 Feb 18]. Available from: https://goo.gl/MEjexy

8. Akpan MR, Ahmed R, Shebl NA, Ashiru-Oredope D. A review of quality measures for assessing the impact of antimicrobial stewardship programs in hospitals. Antibiotics (Basel). 2016:5(1):5.
9. John JF Jr, Fishman NO. Programmatic role of the infectious diseases physician in controlling antimicrobial costs in the hospital. Clin Infect Dis. 1997;24(3):471-85.

10. Anti-Infective Review Panel. Anti-infective guidelines for communityacquired infections. 2012 ed. Toronto (ON): MUMS Guideline Clearinghouse; 2012.

11. Gilbert DN, Eliopoulos GM, Chambers HF, Saag MS, Black D, Freedman DO, et al., editors. The Sanford guide to antimicrobial therapy 2014. 44th ed. Sperryville (VA): Antimicrobial Therapy, Inc; 2014.

Kerry-Anne Hogan, RN, PhD, is Chief Research Officer with the Winchester District Memorial Hospital, Winchester, Ontario. She is also a part-time professor with the University of Ottawa, Ottawa, Ontario.

Mohamed Gazarin, DPharm, is a Clinical Pharmacist with the Winchester District Memorial Hospital, Winchester, Ontario.

Julie Lapenskie, HBHSc, is a Research Assistant with the Winchester District Memorial Hospital, Winchester, Ontario.

Competing interests: None declared.

Address correspondence to:

Dr Kerry-Anne Hogan

Winchester District Memorial Hospital

566 Louise Street

Winchester ON KOC 2KO

e-mail: khogan@wdmh.on.ca

Funding: None received.

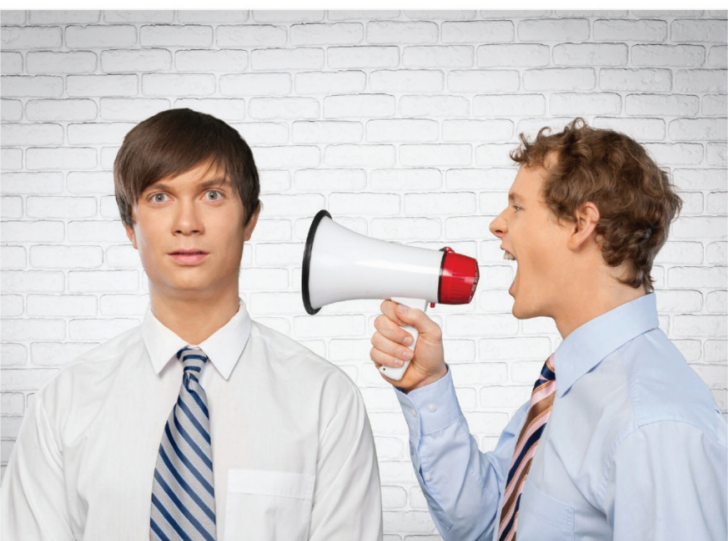

REFER A FRIEND
$\mathrm{D}$

id you know that most of our new members and supporters learn about

CSHP through word of mouth?

Over the years, you have referred hundreds of your friends and colleagues to the Society-THANK YOU!

We'd like to encourage you to keep talking about us!

CSHP offers a referral program that allows you to gain credit that can be applied to publications, continuing education programs, and membership.

* Receive a $\$ \mathbf{1 0}$ credit for referring other members and supporters.

* Receive a $\mathbf{\$ 5}$ credit for referring other students and residents.

New members and supporters will have the opportunity to list you as the person who referred them to CSHP on their application-it's that simple!

For more information, please contact:

Membership Services

Telephone: (613) 736-9733, ext. 222

Email: membershipservices@cshp.ca 